\title{
Metallographic Preparation, Imaging and Analysis of High Purity Refractory Metals
}

\author{
G.M. Lucas* J. Spanos** \\ * Buehler, Ltd. Customer Service Laboratory, 41 Waukegan Road, Lake Bluff, IL 60044 \\ ** Williams Advanced Materials $\backslash$ Pure Tech, 2978 Main Street, Buffalo, New York 14214
}

Webster's New World Dictionary defines "refractory" as \#1 hard to manage; stubborn; obstinate: said of a person or animal. However, what is more pertinent to this topic is definition \# $\mathbf{2}$ resistant to heat, hard to melt or work: said of ores or metals. [1] The term "refractory metals" is used somewhat arbitrarily in the metals industry today, but denotes metals with a very high melting point, $>1900^{\circ} \mathrm{C}$. For the purpose of this paper the periodic table groups of 4, 5, and 6 were chosen: titanium, zirconium, hafnium, vanadium, niobium, tantalum, chromium, molybdenum, and tungsten. The metals used in this study are $99.95 \%$ - $99.999 \%$ pure. High purity refractory metals present unique specimen preparation challenges that must be overcome in order to observe their true structure. Effective preparation and analysis of refractory metals is achieved through the proper sectioning, mounting, polishing, and etching procedures. Once the proper procedures are employed, the prepared surface can be imaged and analyzed accurately.

Because of their tenacious nature, refractory metals are difficult to section without causing heat and mechanical deformation. These metals can range from being ductile to brittle. They are not all hard but they are abrasion resistant. Some use the term "gummy" to describe their resistance to being cut. When sectioning with a high speed saw, a medium particle size, high concentration diamond blade is recommended. It is also helpful to dress the blade more frequently than would normally be done. If an abrasive cut-off saw is being used, choose a wheel with a soft bonding material such as one used for cutting titanium alloys. The problems that may be encountered are abrasive particle embedment and over heating because the wheel may not break down properly. To avoid these problems, cut slowly with equal amounts of coolant on each side of the wheel, applying medium pressure with a pulsing cutting action. Orbital cutters are particularly well suited for sectioning of refractory metals.

The hardest possible compression mounting material should be selected; for example, a thermosetting mineral-filled, epoxy. The temperatures that will be reached in compression mounting will have no effect on the specimen. The reason for the hard mounting material is that many of the polishing steps will be much longer than those on other more common materials. Therefore, the hard mount medium, being a closer match in abrasion resistance to the metals themselves, will enhance edge retention.

If sectioning is done properly a minimal amount of coarse or medium grinding will be necessary. Also, using a rolling abrasive as soon as possible in the grinding/polishing sequence is recommended because of the gentler removal characteristics of rolling abrasives verses fixed abrasives. Fixed abrasives leave a deeper mechanical deformation zone than rolling abrasives when using similar particle sizes. [2] A good basic method to start with is to do initial grinding to remove sectioning deformation with either 320 grit abrasive papers or coarse polish with $15 \mu \mathrm{m}$ diamond on a hard surface. This would be followed by polishing with $9 \mu \mathrm{m}$ diamond slurry on a hard cloth such as a silk 
adhered to a Mylar backing, then $3 \mu \mathrm{m}$ on a similar cloth or one that is slightly softer. The final polishing is achieved on a synthetic cloth such as a polyurethane foam with a colloidal silica, $\mathrm{pH} \sim 9$, with the addition of $30 \%$ hydrogen peroxide for some of the metals.

As shown in figure 2, once a deformation free surface is obtained etching can be performed to reveal the true structure. Figure 3 illustrates the grain size measured by image analysis.

\section{References}

[1] Victoria Neufeldt Editor, Webster's New World Dictionary, Prentice Hall Trade, 1988

[2] George Vander Voort, Metallography, principles and practice, McGraw-Hill New York, 1984

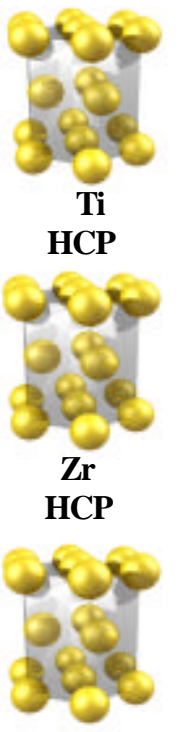

Hf

HCP

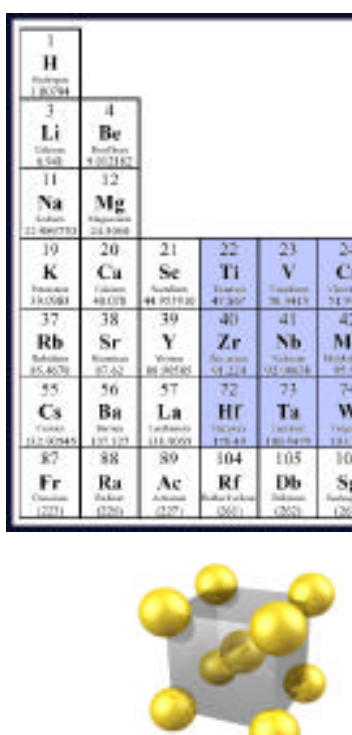

V
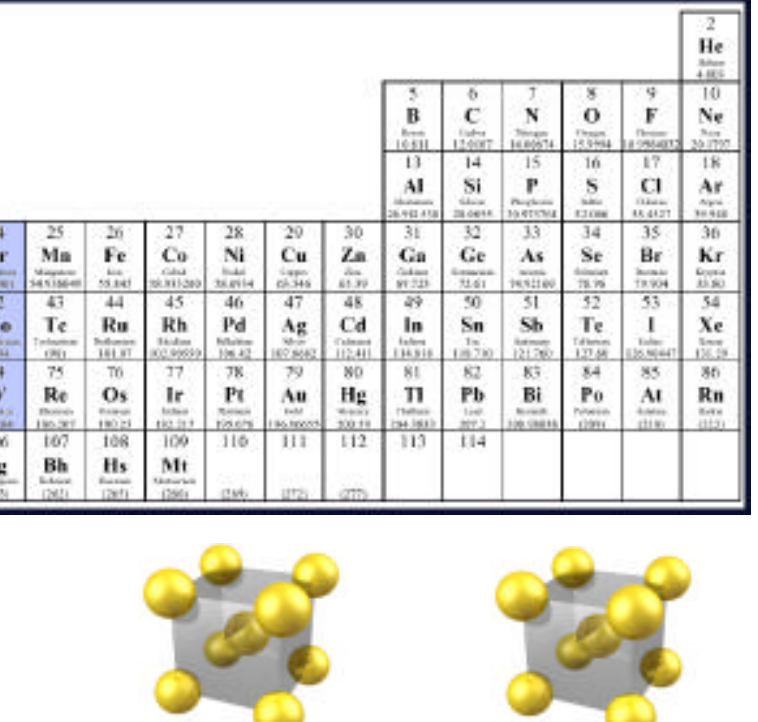

$\mathrm{Nb}$

BCC

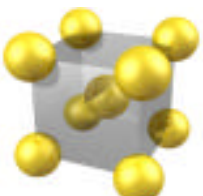

Ta

BCC
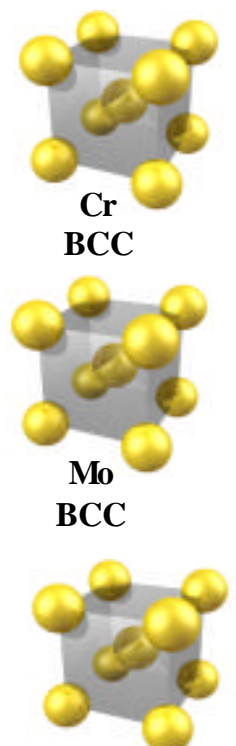

W

BCC

Fig.1. Periodic Table of the Elements and Metals selected for experimentation

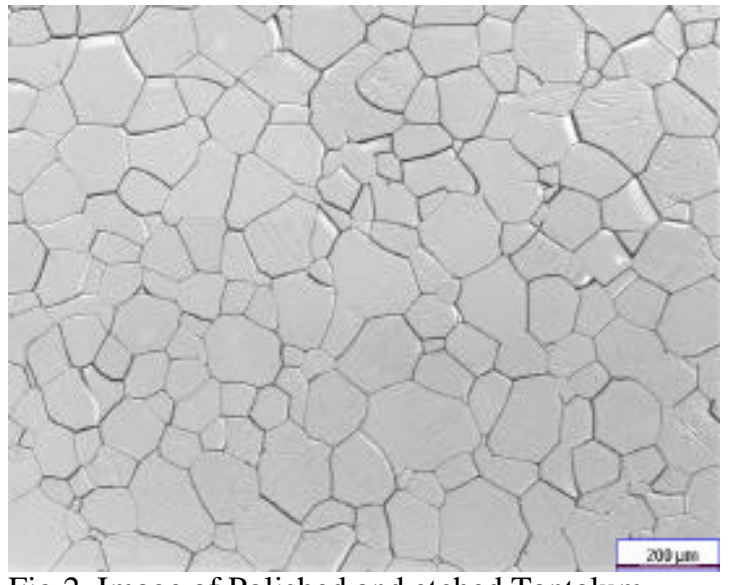

Fig.2. Image of Polished and etched Tantalum

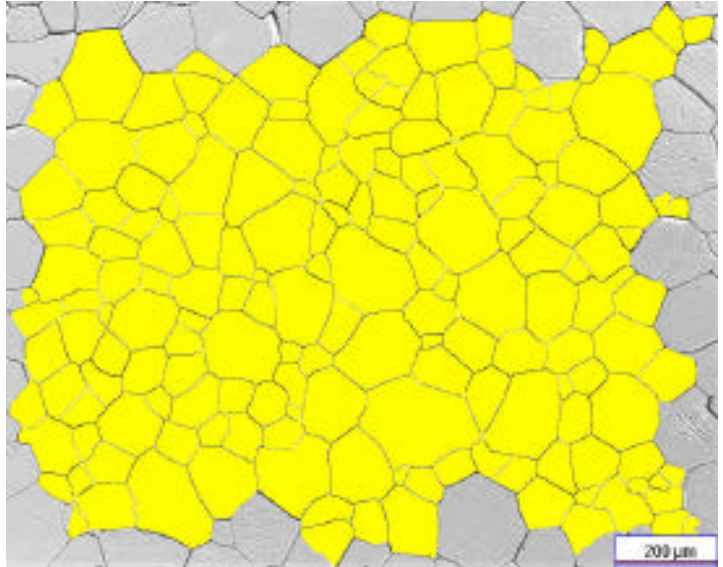

Fig.3. The same image, thresholded for grain size measurement 